\title{
HISTORY
}

\author{
AND \\ DISSECTION OF A CASE,
}

IN WHICH THERE HAD BEEN

\section{DISLOCATION OF THE ANKLE}

WITH

FRACTURE OF THE FIBULA.

BY W: LAWRENCE, ESQ., F.R.S.,

PRESIDENT OF THE SOCIETY.

READ NOVEMBER 22ND, 1831.

ON the 17th of February, 1831, I was desired to see at Bethlem John Simon Steitz, a German, about forty years of age, who had been admitted as a lunatic on that day. He was lame in the left leg in consequence of a fall, which the persons who brought him from a private lunatic establishment represented to have occurred three or four weeks previously. I found him dressed and moving about lamely on the affected limb. The case was dislocation of the ankle with fracture of the fibula. The sole of the foot was turned outwards; the outside of the leg was bent inwards just above the ankle, where the fibula had been broken; and the lower end of the tibia, having lost its proper bearing on the astragalus, formed a large unnatural projection on the inside of the ankle. There was great and obvious deformity of the leg at its lower part, of the ankle and foot, which were red 
and considerably swelled. The patient said that he did not feel, and had not felt, any considerable pain; that no treatment had been employed; and that he had gone about as usual since the accident, but with lameness. Having used considerable force without being able to replace the foot in its proper position, I directed the application of leeches and other means calculated to reduce the swelling. On the following day I succeeded in reducing the dislocation, after which the bandages and splints necessary for confining the foot and broken bone were applied. The patient was kept in bed, and was confined by the right arm and leg, as he frequently loosened and displaced the splints. On those occasions the projection of the tibia was renewed; but the eversion of the sole did not recur. He experienced no uneasiness, and constantly said that he could walk about very well if he were allowed to get up. He was however confined to the recumbent position for six weeks, and not allowed to bear on the foot for a fortnight longer. At the end of another fortnight he had regained the use of the limb so completely, that he needed no support, and could walk without any perceptible lameness. He seemed equally strong on both legs. The sole of the left foot rested flat on the ground; but there was a considerable prominence on the inside of the ankle from unnatural projection of the tibia at its lower end.

J. S. Steitz recovered sufficiently from his mental disorder to be discharged from Bethlem in June: 
he was brought back to the hospital in August, being then in a state of most furious mania, which continued till the beginning of November, when he sunk completely exhausted.

Examination of the foot.-The tibia had partially lost its bearing on the astragalus. The outer half only of its inferior articular surface rested on the latter bone; the inner portion, with the malleolus internus, formed the unnatural prominence on the inside of the ankle. The fibula had been broken just above the external malleolus, and the fracture: had been consolidated by a large formation of bony matter, which had made its lower extremity nearly equal in size to that of the tibia, and had anchylosed it to the latter bone. The superior extremity of the fibular fracture must have rested in the first instance on the outer half of the superior articular surface of the astragalus, and must have rubbed over it in its recently fractured and rough state, as long as the patient continued to use the joint. The asperities however had disappeared, and the consolidated fracture had become covered by a tolerably smooth cartilaginous layer continuous with the articular surfaces of the tibia and of the external malleolus.

The superior convex surface of the astragalus was partially flattened; and the cartilaginous covering was rubbed off it on the outside, where the broken end of the fibula had come in contact 
with it. The bony substance thus denuded was however smooth.

The internal lateral ligament was greatly thickened, and formed a hard fibro-cartilaginous mass, in which some bony matter was deposited. Its attachment to the tibia was nearly as thick as the end of the thumb, and extended to that part of -the inferior surface of the bone which projected over the inner edge of the astragalus.

The ligaments connecting the external malleolus to the foot were unaltered.

The anterior articular convexity of the astragalus was partially raised out of the corresponding cavity of the os naviculare, apparently from the former bone having been subjected to unusual pressure on the back and outer part of its upper surface, in consequence of the altered bearing of the leg.

The nearly complete recovery of the joint after so serious an injury must be considered an unexpectedly fortunate occurrence, when there had been not only a complete neglect of treatment, but also continued exertion of the injured part for three or four weeks.

In consequence of the partial separation of the tibia from the astragalus, the weight of the body must have been transmitted to the latter bone partly by the fibula, of which the broken end rested upon it. 
62 CASE OF DISLOCATION OF THE ANKLE.

The copious deposition of new bone, by which the fracture was consolidated and anchylosed to the tibia, gave to the part in question the additional strength which it required under these altered circumstances. The great thickening of the internal lateral ligament was an analogous provision on the opposite side of the joint against that eversion of the sole which might have been expected after such a dislocation. 